\title{
A case of vertebral artery duplication at the level of atlas: anatomical description
}

\author{
Maciej K. Ciołkowski • Paweł Krajewski • \\ Bogdan Ciszek
}

Received: 11 October 2013/Accepted: 4 April 2014/Published online: 21 April 2014

(C) The Author(s) 2014. This article is published with open access at Springerlink.com

\begin{abstract}
Purpose To present case of vertebral artery duplication surrounding the posterior arch of atlas.

Methods The vertebral artery variation was encountered during dissection of specimen from the body of a 62-yearold man.

Results The vertebral artery formed a circle located extraduraly, surrounding the posterior arch of atlas vertebra. Topography and morphometry of the finding is described. Embryology and clinical significance based on literature review are discussed.

Conclusions Precise diagnostic imaging is necessary before surgical interventions at the level of cranio-spinal junction in order to avoid conflict with rare vascular variations.
\end{abstract}

Keywords Vertebro-basilar circulation - Vertebral artery fenestration - Anatomical variation · Developmental abnormality $\cdot$ Cranio-spinal junction

M. K. Ciołkowski ( $₫) \cdot$ B. Ciszek

Department of Descriptive and Clinical Anatomy, Centre of Biostructure Research, Medical University of Warsaw, Chałubińskiego 5, 02-004 Warsaw, Poland

e-mail: maciej.ciolkowski@wum.edu.pl

M. K. Ciołkowski

Department of Neurosurgery, Children's Memorial Health Institute, Warsaw, Poland

P. Krajewski

Department of Forensic Medicine, Centre of Biostructure Research, Medical University of Warsaw, Warsaw, Poland

B. Ciszek

Department of Neurosurgery, Prof. Bogdanowicz Children Hospital, Warsaw, Poland

\section{Introduction}

Course of the vertebral artery and location of its branches may significantly differ from the classic type. For example, according to Argenson et al. [1] in $10.1 \%$ of cases the vertebral artery enters the transversary foramina at levels other to C6. Yamazaki et al. [2] found the vertebral artery passing between the posterior arch of atlas and arch of axis in $8 \%$ of patients operated for diseases of cranio-vertebral junction. In $2 \%$ of these patients the vertebral artery formed a loop around the posterior arch of atlas.

\section{Case report}

In the material of 50 specimens of suboccipital region a case of unilateral atypical branching pattern of atlantic part of the vertebral artery was observed in the specimen from the body of a 62-year-old man who died after head trauma. All the specimens were collected during routine forensic autopsies, injected with coloured gelatine and fixed in formaldehyde solution. The vertebral arteries of the discussed specimen had diameter of $4.61 \mathrm{~mm}$ on the left and $4.50 \mathrm{~mm}$ on the right measured just behind the dura. Microsurgical dissection of the atlantic part of the left vertebral artery (V3) revealed following branches: two articular (with diameters of 0.52 and $0.42 \mathrm{~mm}$ ), two muscular (with diameters 1.74 and $1.80 \mathrm{~mm}$ ), posterior spinal artery of extradural origin (with diameter of $0.82 \mathrm{~mm}$ ). Additionally, at the anterior edge of the bony groove on the posterior arch of atlas the vertebral artery gave of a large branch measuring $3.32 \mathrm{~mm}$ in diameter at this point. This branch coursed epiduraly downwards between the anterior and posterior roots of the first cervical nerve, then together 

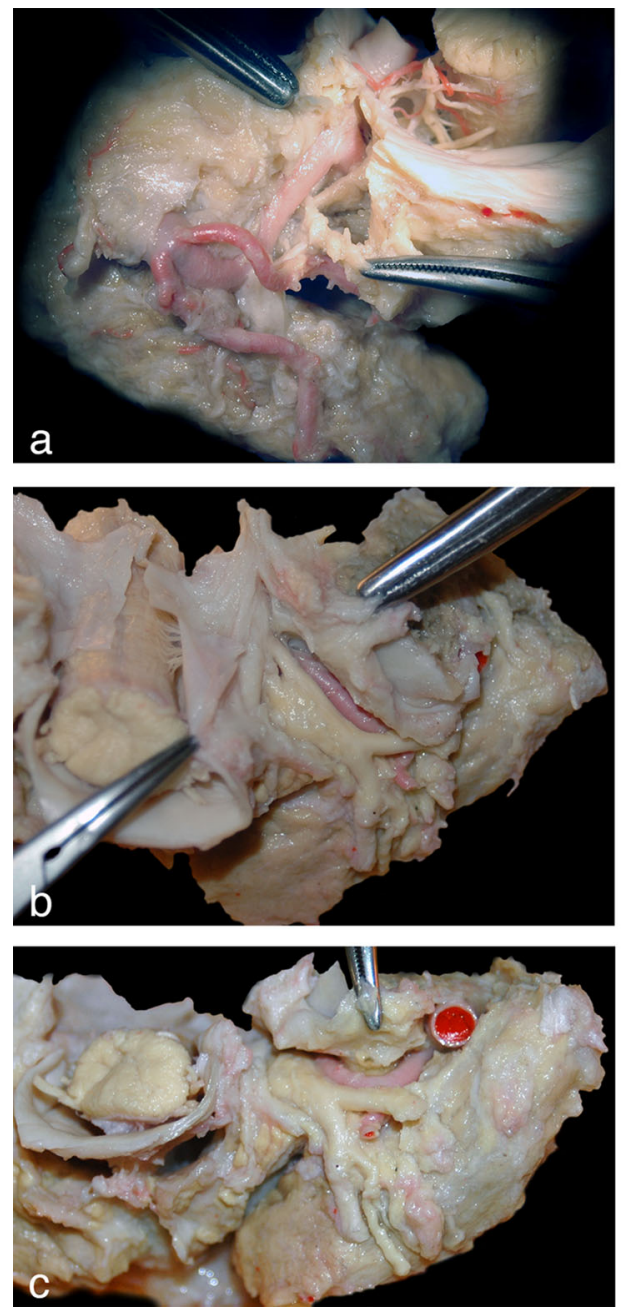

Fig. 1 Dissections of the discussed specimen. a Superior-posterior view: the posterior atlanto-occipital membrane $(P A O M)$ and dura $(D)$ were cut to visualise the vertebral artery-its atlantic (V3) segment continuous with the intradural one (V4). The inferior arm of the arterial ring (arrow) branches of the $\mathrm{V} 3$ and runs downwards epiduraly, anterior to the dorsal root and ganglion of the first cervical nerve $(\operatorname{drg} C 1)$. The $\mathrm{V} 3$ gives of also deep and superficial muscular branches $(d m b, s m b)$ and posterior spinal artery (PSA). OCIM obliquus capitis inferior muscle, $X I$ spinal root of the accessory

with the second cervical nerve made a loop between the arches of the atlas and axis vertebrae and just posterior and lateral to the lateral atlanto-axial joint it anastomosed with the terminal segment of transversary part of the veretebral artery (V2) within the $\mathrm{C} 1$ transversary foramen having diameter of $2.86 \mathrm{~mm}$ (Fig. 1). The arterial segment passing between the arches of atlas and axis was giving off arterioles to the lateral atlanto-axial joint, a branch supplying the second spinal nerve $(0.60 \mathrm{~mm}$ in diameter $)$ and a muscular branch (1.24 $\mathrm{mm}$ in diameter). Otherwise the specimen showed usual anatomy of soft and bony structures.
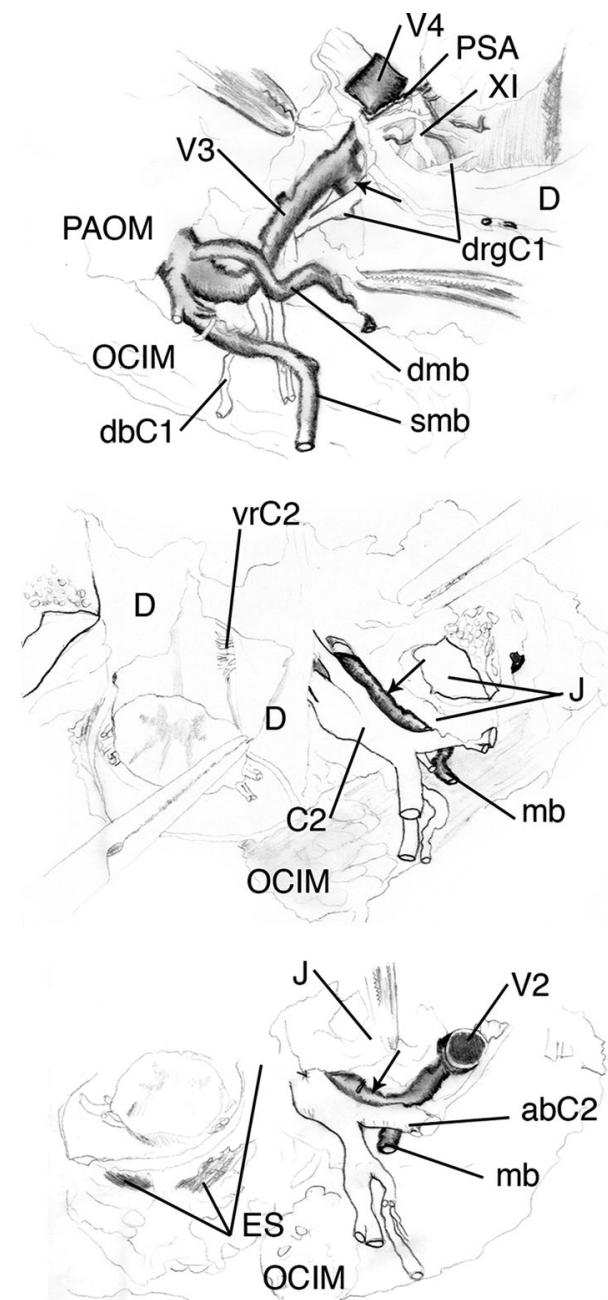

nerve, $d b C 1$ dorsal branch of the first cervical nerve (suboccipital nerve). b Anterior-inferior view. c Inferior view: course of the inferior arm of the discussed arterial ring is shown in relationship with the lateral atlanto-axial joint ( $J$ inferior articular surface of atlas and articular capsule are visible) and with the second cervical nerve $(C 2)$ and its anterior branch ( $a b C 2), v r C 2$ ventral roots of $\mathrm{C} 2$ nerve, $m b$ muscular branch, $V 2$ terminal part of the transversary segment of vertebral artery, $E S$ epidural space

\section{Discussion}

The loop of vertebral artery encircling the posterior arch of atlas vertebra was first described by Kadyi in 1888 [3] in his monograph on vascularisation of spinal cord and, earlier, by Herberg in 1845 as quoted by him. There is significant number of papers discussing fenestrations and duplications of the vertebral artery based on angiographic imaging examinations [4-9]. Lasjaunias [5, 10] basing on his experience with classic angiography in numerous anatomical variants and abnormalities and earlier embryological studies of Padget [11], proposed a schematic 'grid' 
model explaining embryology of normal and abnormal vertebro-basilar system. The transversary segment (V2) of vertebral artery originates from sequence of primitive anastomoses between the cervical intersegmental arteries, while the horizontal loop of atlantic segment (V3) of vertebral artery originates from the proatlantic intersegmental artery located between the skull and spine. If the first cervical intersegmental artery does not disappear during development, the vertebral artery runs between arches of the atlas and axis vertebrae. If both proatlantic and first cervical intersegmental arteries persist with their anastomoses, the 'window' within the vertebral artery develops, encircling the posterior arch of atlas, like in the case presented here. Lasjaunias [5] differentiated the true fenestration, which is a local split of vascular lumen, from the duplication, where vessels have different course before they fuse again. Like in our case, arms of local duplication have origin in different embryonic vascular trunks. Such atypical development may be accidental, but may have also complex genetic basis, as such variations in the vertebral artery course are more frequently seen in patients with Down syndrome examined because of atlanto-axial subluxation [12].

To our best knowledge, there are only two cases of vertebral artery passing between the atlas and axis together with the $\mathrm{C} 2$ spinal nerve described in contemporary literature and verified by anatomical dissection [7, 13, 14]. In these cases vertebral artery pierced the dura below the level of atlas vertebra, but the $\mathrm{C} 1$ transversary foramen and suboccipital space contained no artery. The authors mentioned above in cases of vertebral artery duplication usually describe the arterial branch visualised in the vertebral canal as located intraduraly. Reliable assessment of the true location of the artery in the vertebral canal seems to be hardly possible if just classic angiographic pictures are available. Modern diagnostic tools like CT and MR imaging with three-dimensional reconstructions give much more complete information and are necessary for good preoperative assessment in every case. The variation presented here, however rare, may seriously complicate $\mathrm{C}_{0}-\mathrm{C}_{1}-\mathrm{C}_{2}$ stabilisation procedures or make vertebral artery transposition during far lateral/transcondylar approaches very difficult.

\section{Conclusion}

In the case presented here the whole ring of duplicated vertebral artery surrounding the posterior arch of atlas was located extraduraly. Similar variations of vertebral artery enlarge the risk of serious complications of surgical and percutaneous interventions, like blockades or $\mathrm{C} 1-\mathrm{C} 2$ space puncture. Surgeons performing laminectomies or instrumental stabilisations should be aware of possible epidural location of atypical vertebral artery.

Conflict of interest None of the authors has any potential conflict of interest.

Open Access This article is distributed under the terms of the Creative Commons Attribution License which permits any use, distribution, and reproduction in any medium, provided the original author(s) and the source are credited.

\section{References}

1. Argenson C, Francke JP, Sylla S, Dintimille H, Papasian S, di Marino V (1980) The vertebral arteries (segments V1 and V2). Anatomia Clinica 2:29-41

2. Yamazaki M, Okawa A, Furuya T, Sakuma T, Takahashi H, Kato K, Fujiyoshi T, Mannoji C, Takahashi K, Koda M (2012) Anomalous vertebral arteries in the extra- and intraosseous regions of the craniovertebral junction visualised by 3D CT angiography: analysis of 100 consecutive surgical cases and review of the literature. Spine 37(22):E1389-E1397

3. Kadyi H (1889) Über die Blutgefässe des Menschlichen Rückenmarkes. Verlag von Gubrynowicz \& Schmidt, Lemberg (Polish edn: Lwów 1888)

4. Kowada M, Yamaguchi K, Takahashi H (1972) Fenestration of the vertebral artery with a review of 23 cases in Japan. Radiology 103(5):343-346

5. Lasjaunias P, Braun JP, Hasso AN, Moret J, Manelfe C (1980) True and false fenestration of the vertebral artery. J Neuroradiol 7:157-166

6. Rieger P, Huber G (1983) Fenestration and duplicate origin of the left vertebral artery in angiography. Report of three cases. Neuroradiology 25:45-50

7. Tokuda K, Miyasaka K, Abe H, Abe S, Takei H, Sugimoto S, Tsuru M (1985) Anomalous atlantoaxial portions of vertebral and posterior inferior cerebellar arteries. Neuroradiology 27:410-413

8. Sanders WP, Sorek PA, Mehta BA (1993) Fenestration of intracranial arteries with special attention to associated aneurysms and other anomalies. AJNR Am J Neuroradiol 14:675-680

9. Siclari F, Burger IM, Fasel JHD, Gailloud P (2007) Developmental anatomy of the distal vertebral artery in relationship to variants of the posterior and lateral spinal arterial systems. AJNR Am J Neuroradiol 28:1185-1190

10. Lasjaunias P (1983) Craniofacial and upper cervical arteries: Collateral circulations and angiographic protocols. Williams and Wilkins, Baltimore, pp 15-27

11. Padget DH (1954) Designation of the embryonic intersegmental arteries in reference to the vertebral artery and subclavian stem. Anat Rec 119(3):349-356

12. Yamazaki M, Okawa A, Hashimoto M, Aiba A, Someya Y, Koda M (2008) Abnormal course of the vertebral artery at the craniovertebral junction in patients with down syndrome visualized by three-dimensional CT angiography. Neuroradiology 50:485-490

13. Takahashi K (1963) A case of the vertebral artery not passing through the foramen transversarium of the atlas. Sapporo Med J $24: 250-252$

14. Abe K (1968) A rare abnormal case of the vertebral artery showing no passing through the foramen transversarium of the atlas. Acta Anatomica Nipponica 43:393-394 